\title{
Tuning Successive Linear Programming to Solve AC Optimal Power Flow Problem for Large Networks
}

\author{
Sayed Abdullah Sadat ${ }^{\mathrm{a}}$, Mostafa Sahraei-Ardakani ${ }^{\mathrm{a}}$ \\ ${ }^{a}$ Department of Electrical and Computer Engineering, University of Utah, Salt Lake City, Utah,
}

\begin{abstract}
Successive linear programming (SLP) is a practical approach for solving large-scale nonlinear optimization problems. Alternating current optimal power flow (ACOPF) is no exception, particularly the large size of real-world networks. However, in order to achieve tractability, it is essential to tune the SLP algorithm presented in the literature. This paper presents a modified SLP algorithm to solve the ACOPF problem, specified by the U.S. Department of Energy's (DOE) Grid Optimization (GO) Competition Challenge 1, within strict time limits. The algorithm first finds a near-optimal solution for the relaxed problem (i.e., Stage 1). Then, it finds a feasible solution in the proximity of the near-optimal solution (i.e., Stage 2 and Stage 3). The numerical experiments on test cases ranging from 500-bus to 30,000-bus systems show that the algorithm is tractable. The results show that our proposed algorithm is tractable and can solve more than $80 \%$ of test cases faster than the well-known Interior Point Method while significantly reduce the number of iterations required to solve ACOPF. The number of iterations is considered an important factor in the examination of tractability which can drastically reduce the computational time required within each iteration.
\end{abstract}

Keywords: optimal power flow, successive linear programming, power systems operation, nonlinear programming, tractable algorithms

\section{Introduction}

Power system operation aims to deliver power to customers in a reliable and cost-effective manner. Optimal and reliable power dispatch is an optimization problem, which in its original form is referred to as "alternating current optimal power flow" (ACOPF) problem as described in [1, 2]. ACOPF is a nonlinear optimization problem with non-convex constraints, making it NP-hard [3].

The computational challenge is also significant because of the large size of real-world networks. The power grid in the United States is the largest machine ever built by humans, with hundreds-of-thousands of buses, transmission elements, and generators in three synchronous interconnections. According to [4], the aggregate sum of annual retail transactions in the US power grid now exceeds USD 400 billion. Clearly, a costeffective operation is important due to the economic impacts of this crucial infrastructure.

Because of the difficulty of solving the ACOPF problem, the majority of the grid operators use some form of a direct current optimal power flow (DCOPF) approximation as described by [5]. The DCOPF linearization shown in a market problem by [6, 7] is based on a few assumptions that convert the nonlinear ACOPF problem to a linear formulation. Although the implementations enable solving optimal power flow within the practical time constraints, rough approximations of voltage and reactive power flow are applied, requiring the application of conservative transmission limits. This can result in an inefficient and sub-optimal operation of the grid.

According to a staff report by the U.S. Federal Energy Reg- ulatory Commission (FERC) [1], the differences between a DCOPF and a more-efficient operations that may be achieved with an ACOPF may incur 10\% additional cost due to the sub-optimal operation of the grid. In the United States, the economic costs represent tens of billions of dollars each year. Moreover, a good solution to ACOPF is critical to many complex decision-making processes, including consideration of trade-offs between active and reactive power flows, as mentioned by [8, 9, 10, 11]. Thus, developing a method that efficiently solves the ACOPF problem can have a significant economic impact.

The Interior-Point Line Search method is a powerful algorithm for solving large-scale nonlinear optimization problems [12, 13]. IPOPT efficiently implements the Interior-Point Line Search filter method, which is typically used as a benchmark by most of the recent work on ACOPF algorithms [14, 3, 15]. Thus, we compare the performance of the IPOPT solver with our algorithm to show the effectiveness of our proposed algorithm in solving ACOPF problem.

The rest of the paper is organized as follows. A literature review is presented in Section 2. Section 3 introduces the ACOPF formulation. In Section 4, we present our methodology. In section 5, we describe the implementation of our proposed algorithm. Section 6 presents the results of our numerical experiments on the proposed method and demonstrates its effectiveness on various ARPA-E networks ranging from 500-bus to 30,000-bus systems. Finally, Section 7 concludes this paper. 


\section{Literature Review}

Over the past few decades, many methods have been proposed to solve the ACOPF problems. However, the majority of them were not focused on solving large-scale problems within the industry time standards. For example, [16] and [17] discuss convex relaxation and analyze the structure of an ACOPF problem. J. Lavaei et al. [18], R. Madani et al. [19], and X. Bai et al. [20] propose semidefinite programming (SDP) methods for solving ACOPF problems. However, these methods were tested on systems only as large as 300 buses. Although, relaxations are useful for verification of global optimality, they mainly suffer from two drawbacks. first, SDP relaxation even with chordal extension is computationally intractable [21]. second, there is no sufficient condition known for exact semidefinite relaxation for general mesh networks yet [22]. Others have attempted to solve the non-linear ACOPF problem directly. A. F. Attia [23], G. Torres et al. [24] and [25] proposed an interior-point method approach to directly solve ACOPF problems. However, interior-point methods are only suitable for small to medium size problems.

On the other hand, active-set methods are known to be more suitable for large-scale problems. Active-set methods are iterative methods that solve a sequence of subproblems, either quadratic or linear subproblem depending on the method. The goal of the method is to predict the active set, the set of constraints that are satisfied at the solution of the problem [26]. Successive linear programming (SLP) is an active-set method that has recently attracted attention in power systems. According to R O'Neil et al. [27], the SLP method takes advantage of the linear representation of network flows, which provides easy integration into existing energy and market management systems that may require linear "shift factor" data. It also leverages the deployment of off-the-shelf commercial linear programming solvers as described by [8]. However, the performance of the SLP method, in terms of convergence quality, objective value, and computational performance, largely depends on the initialization of the SLP algorithm as shown by [28, 29, 30].

A. Castillo et al. [8] applies Successive Linear Programming to solve the ACOPF problem. The paper prefers current voltage in rectangular form over other formulations of ACOPF. It adopts a heuristic approach for controlling the step size. The algorithm's main focus in [8] is to obtain an AC feasible solution to the ACOPF problem. Z. Yang et al. [31] present a comparison of different linearization techniques for nonlinear constraints in the ACOPF, such as DC model, first-order Taylor Series approximation, and preservation of second-order terms. The comparison demonstrates the impact of different linearization techniques on their accuracy of representing the ACOPF model in a canonical form. Z. Yang et al. [32, 33] introduce an iterative approach to solve an ACOPF problem. Their methods, similar to the work by [31], are based on the polar form of state variables. This work is focused on obtaining a feasible solution and uses constraints reduction techniques similar to [8]. The linearization techniques mentioned in [32] with an error handling approach make this work distinctive. However, it does not present any step control algorithm. P. Fortenbacher et al. [34] offers a non-iterative linear programming (LP)/quadratic programming (QP) based solution approach for an optimal power flow problem. The paper focuses on modeling absolute loss approximations and adding them as linear constraints to the ACOPF problem. In one of the proposed approaches, integer variables are used for loss models, which can make the problem computationally intractable for large networks.

A. A. Mohamed et al. [35] presents a line-wise optimal power flow formulation solved using successive linear programming method. The authors claim better performance of their proposed method over MATPOWER solver and bus-wise ACOPF formulation. It also uses squares of nodal voltage magnitudes as a set of control variables. It solves a set of nonlinear equations using the Newton-Raphson method before solving each linear programming sub-problem. The work also suggests the choice of a step-size based on experience with each network. The paper initially discusses local optimality versus global optimality. However, neither first-order nor second-order optimality conditions are part of the termination criteria. This indicates that the proposed work searches for a feasible solution rather than a locally optimal solution. L. Sampath et al. [36] proposes solving an ACOPF problem in polar form using a trust-region sequential linear programming method. The method adapts the first-order optimality condition from necessary Karush-Kuhn-Tucker (KKT) conditions proposed by [37], and therefore, local optimality is sought by the algorithm. The work by [8] is used as a benchmark by [36] to compare the proposed algorithm's performance with other methods. S. Mhanna et al. [38] presents an SLP algorithm by constructing supporting hyperplanes and halfspaces in LP subproblem to match its performance with interior point method for test cases smaller than 4000 buses.

The past work presents a great deal of progress in solving ACOPF problems. However, the main challenge is yet to be completely resolved, which is solving ACOPF problem within the industry time standards, particularly for large-scale problems. The main drawback of the methods discussed in this Section is that they lose tractability when applied to large-scale problems [20, 29].

\section{The ACOPF Formulation}

In an ACOPF problem, the most challenging constraints are the equations that represent transmission branches. There are different approaches to model a transmission branch in a power system. The most commonly used approach is called the $\pi$ model. In this paper, we apply the $\pi$-model to represent the transmission branches in our formulations as shown in Fig. 1. The notation conventions used throughout this paper are described in Appendix A

Let $y_{i j}^{n e t}=y_{i j}^{M}+\left(y_{i j}+y_{i j}^{s}\right) \cdot\left|\tau_{i j}\right|^{2}, y_{j i}^{n e t}=y_{i j}+y_{i j}^{s}$, and $2 \mathcal{K}=$ $\{i, j\} \in \mathcal{K} \cup\{j, i\} \in \mathcal{K}$. The admittance matrix can be written as shown in (1), where our formulation treats $\tau_{i j}$ as constant parameters. 


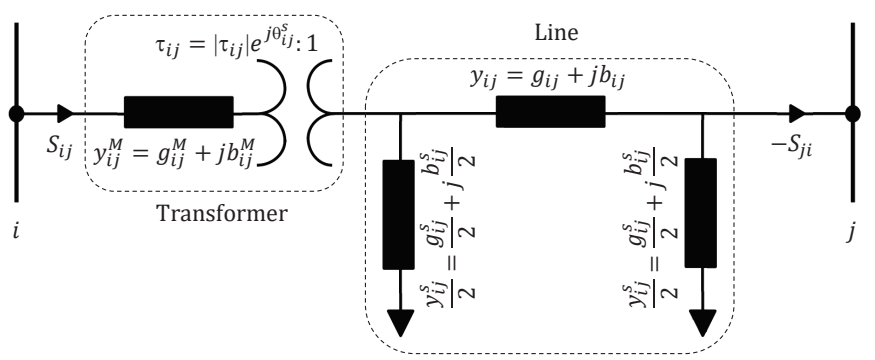

Figure 1: A general representation of a $\pi$-model of a transmission branch. If a branch is only a transmission line then $\tau_{i j}=1$ and $y_{i j}^{M}=0$. If a branch is only a transformer, then all the line parameters are zero. Thus, the figure represents a general model for a transmission branch representing any transmission element by simply adjusting the values of the given parameters [39].

$$
Y_{i i}=\sum_{\substack{k=1 \\ k \neq i}}^{\mathcal{K}_{i}^{f}} y_{i k}^{\text {net }}+\sum_{\substack{k=1 \\ k \neq i}}^{\mathcal{K}_{i}^{t}} y_{k i}^{\text {net }}, \quad Y_{i j}=-y_{i j} \cdot \tau_{i j}^{*}, \quad Y_{j i}=-y_{i j} \cdot \tau_{i j}
$$

An ACOPF problem can be formulated as an optimization problem, and its objective function depends on the objectives of the ACOPF problem. In this paper, we assume the objective function is to minimize the generators real power costs, which are usually represented as a quadratic function of each generator's real power output. A simple ACOPF problem is shown in (2).

$$
\min \sum_{g \in \mathcal{G}} c_{2 g} \cdot\left(\mathfrak{R}\left[s_{g}\right]\right)^{2}+c_{1 g} \cdot \mathfrak{R}\left[s_{g}\right]+c_{0 g}
$$

s.t. $\quad S_{i j}=v_{i} \cdot i_{i j}^{*}=v_{i} \cdot\left(y_{i j}^{n e t} \cdot v_{i}+Y_{i j} \cdot v_{j}\right)^{*}, \forall\{i, j\} \in 2 \mathcal{K}$.

$$
\sum_{g \in \mathcal{G}_{n}} s_{g}-\sum_{d \in \mathcal{D}_{n}} s_{d}=\sum_{k \in \mathcal{K}_{n}^{f}} S_{n k}+\sum_{k \in \mathcal{K}_{n}^{t}} S_{k n}+Y_{n}^{L} \cdot\left|v_{n}\right|^{2}, \forall n \in \mathcal{N} .
$$

$$
\begin{aligned}
& V_{n}^{\text {Min }} \leq\left|v_{n}\right| \leq V_{n}^{\text {Max }}, \forall n \in \mathcal{N} . \\
& \left|S_{i j}\right| \leq I_{i j}^{\text {Max }} \cdot\left|v_{n}\right|, \forall\{i, j\} \in 2 \mathcal{K} . \\
& s_{g}^{\text {Min }} \leq s_{g} \leq s_{g}^{\text {Max }}, \forall g \in \mathcal{G} .
\end{aligned}
$$

To separate the real and imaginary parts of (2), we use parameters and variables in rectangular form as shown in (3)

$$
\begin{gathered}
\min \quad \sum_{g \in \mathcal{G}} c_{2 g} \cdot p_{g}^{2}+c_{1 g} \cdot p_{g}+c_{0 g} \\
\text { s.t. } \quad P_{i j}=g_{i j}^{n e t} \cdot\left[\left(v_{i}^{r}\right)^{2}+\left(v_{i}^{i}\right)^{2}\right]+G_{i j} \cdot\left(v_{i}^{r} \cdot v_{j}^{r}+v_{i}^{i} \cdot v_{j}^{i}\right) \\
-B_{i j} \cdot\left(v_{i}^{r} \cdot v_{j}^{i}-v_{i}^{i} \cdot v_{j}^{r}\right), \forall\{i, j\} \in 2 \mathcal{K} . \\
Q_{i j}=-b_{i j}^{n e t} \cdot\left[\left(v_{i}^{r}\right)^{2}+\left(v_{i}^{i}\right)^{2}\right]-B_{i j} \cdot\left(v_{i}^{r} \cdot v_{j}^{r}+v_{i}^{i} \cdot v_{j}^{i}\right) \\
-G_{i j} \cdot\left(v_{i}^{r} \cdot v_{j}^{i}-v_{i}^{i} \cdot v_{j}^{r}\right), \forall\{i, j\} \in 2 \mathcal{K} . \\
\sum_{g \in \mathcal{G}_{n}} p_{g}-\sum_{d \in \mathcal{D}_{n}} p_{d}=\sum_{k \in \mathcal{K}_{n}^{f}} P_{n k}+\sum_{k \in \mathcal{K}_{n}^{t}} P_{k n} \\
+G_{n}^{L} \cdot\left[\left(v_{n}^{r}\right)^{2}+\left(v_{n}^{i}\right)^{2}\right], \forall n \in \mathcal{N} .
\end{gathered}
$$

$$
\begin{aligned}
& \sum_{g \in \mathcal{G}_{n}} q_{g}-\sum_{d \in \mathcal{D}_{n}} q_{d}=\sum_{k \in \mathcal{K}_{n}^{f}} Q_{n k}+\sum_{k \in \mathcal{K}_{n}^{t}} Q_{k n} \\
& \quad-B_{n}^{L} \cdot\left[\left(v_{n}^{r}\right)^{2}+\left(v_{n}^{i}\right)^{2}\right], \forall n \in \mathcal{N} . \\
& V_{n}^{\text {Min }} \leq\left|v_{n}^{r}+j v_{n}^{i}\right| \leq V_{n}^{\text {Max }}, \forall n \in \mathcal{N} . \\
& \left|P_{i j}+j Q_{i j}\right| \leq I_{i j}^{\text {Max }} \cdot\left|v_{n}^{r}+j v_{n}^{i}\right|, \quad \forall\{i, j\} \in 2 \mathcal{K} . \\
& p_{g}^{\text {Min }} \leq p_{g} \leq p_{g}^{\text {Max }}, \forall g \in \mathcal{G} . \\
& q_{g}^{\text {Min }} \leq q_{g} \leq q_{g}^{\text {Max }}, \forall g \in \mathcal{G} .
\end{aligned}
$$

(3f) and (3g) have absolute values, and to address it, we multiply each side with its conjugate to obtain a square of the equations as shown in (4).

$$
\begin{array}{ll}
\min \quad & \sum_{g \in \mathcal{G}} c_{2 g} \cdot p_{g}^{2}+c_{1 g} \cdot p_{g}+c_{0 g} \\
\text { s.t. } \quad & P_{i j}=g_{i j}^{n e t} \cdot\left[\left(v_{i}^{r}\right)^{2}+\left(v_{i}^{i}\right)^{2}\right]+G_{i j} \cdot\left(v_{i}^{r} \cdot v_{j}^{r}+v_{i}^{i} \cdot v_{j}^{i}\right) \\
& -B_{i j} \cdot\left(v_{i}^{r} \cdot v_{j}^{i}-v_{i}^{i} \cdot v_{j}^{r}\right), \forall\{i, j\} \in 2 \mathcal{K} . \\
& Q_{i j}=-b_{i j}^{n e t} \cdot\left[\left(v_{i}^{r}\right)^{2}+\left(v_{i}^{i}\right)^{2}\right]-B_{i j} \cdot\left(v_{i}^{r} \cdot v_{j}^{r}+v_{i}^{i} \cdot v_{j}^{i}\right) \\
& -G_{i j} \cdot\left(v_{i}^{r} \cdot v_{j}^{i}-v_{i}^{i} \cdot v_{j}^{r}\right), \forall\{i, j\} \in 2 \mathcal{K} . \\
& \sum_{g \in \mathcal{G}_{n}} p_{g}-\sum_{d \in \mathcal{D}_{n}} p_{d}=\sum_{k \in \mathcal{K}_{n}^{f}} P_{n k}+\sum_{k \in \mathcal{K}_{n}^{t}} P_{k n} \\
& +G_{n}^{L} \cdot\left[\left(v_{n}^{r}\right)^{2}+\left(v_{n}^{i}\right)^{2}\right], \forall n \in \mathcal{N} . \\
& \sum_{g \in \mathcal{G}_{n}} q_{g}-\sum_{d \in \mathcal{D}_{n}} q_{d}=\sum_{k \in \mathcal{K}_{n}^{f}} Q_{n k}+\sum_{k \in \mathcal{K}_{n}^{t}} Q_{k n} \\
& \left(V_{n}^{\text {Min }}\right)^{2} \leq\left[\left(v_{n}^{r}\right)^{2}+\left(v_{n}^{i}\right)^{2}\right] \leq\left(V_{n}^{M a x}\right)^{2}, \forall n \in \mathcal{N} . \\
& P_{i j}^{2}+Q_{i j}^{2} \leq\left(I_{i j}^{M a x}\right)^{2} \cdot\left[\left(v_{n}^{r}\right)^{2}+\left(v_{n}^{i}\right)^{2}\right], \forall\{i, j\} \in 2 \mathcal{K} . \\
& p_{g}^{\text {Min }} \leq p_{g} \leq p_{g}^{\text {Max }}, \forall g \in \mathcal{G} . \\
& q_{g}^{\text {Min }} \leq q_{g} \leq q_{g}^{\text {Max }}, \forall g \in \mathcal{G} .
\end{array}
$$

The optimization problem shown in (4) is the representation of the ACOPF problem used in our proposed algorithm. The choice of the formulation is based on the work described in [40], which describes rectangular voltage models as more suitable form of ACOPF compared to other forms for the convexification of ACOPF.

\section{Methodology}

Our proposed sequential linear programming algorithm is an approach for solving an ACOPF problem based on activeset methods. Active-set methods reduce search complexity by identifying the most influential constraints in nonlinear optimization problems that need to be watched, while finding the solution [41]. Active-set methods are known to be more sensitive to initialization compared to nonlinear interior-point methods and can be easily exploited for time-series solution of ACOPF [29, 30]. Therefore, they are more appropriate and computationally efficient for time-series solution of ACOPF.

In our proposed algorithm, the ACOPF problem is linearized as an LP sub-problem and it is iteratively solved until the violations in the original nonlinear ACOPF problem are within 
an acceptable tolerance. The main reasons we chose SLP over other methods include:

1. The ability to utilize commercial LP solvers, which are remarkably fast and reliable [38];

2. The sensitivity to initialization, where a better initialization significantly reduces the computational burden [28]. The sensitivity to initialization particularly helps in this application, because we can easily obtain DCOPF solution and utilize it as an initialization for the SLP algorithm;

3. Locational Marginal Prices (LMP), which are the linchpin of electricity markets, are easier to obtain using LP;

4. The system operators and the existing energy management systems (EMS) prefer linearized methods and therefore, SLP can easily integrate with existing EMS systems [5. 42].

To solve an ACOPF problem, we first need to obtain its linear programming sub-problem. To do so, we first linearize the objective function eq. (4a) using piecewise linear interpolation, as described by [43]. In addition, we also need to address the nonlinearities in (4b) - 4g) using first-order Taylor series approximation. The LP sub-problem is shown in (5).

$$
\begin{aligned}
& \text { minimize } \sum_{g \in \mathcal{G}} c_{g} \\
& \text { subject to } \quad c_{g}=\sum_{h \in \mathcal{H}_{g}} c_{g h} \cdot t_{g h}, \forall g \in \mathcal{G} \text {. } \\
& p_{g}=\sum_{h \in \mathcal{H}_{g}} p_{g h} \cdot t_{g h}, \forall g \in \mathcal{G} . \\
& \sum_{h \in \mathcal{H}_{g}} t_{g h}=1, \forall g \in \mathcal{G} . \\
& t_{g h} \geq 0, \forall g \in \mathcal{G}, h \in \mathcal{H}_{g} \\
& P_{i j}=\frac{\partial P_{i j}}{\partial \hat{v}_{i}^{r}} \cdot v_{i}^{r}+\frac{\partial P_{i j}}{\partial \hat{v}_{i}^{i}} \cdot v_{i}^{i}+\frac{\partial P_{i j}}{\partial \hat{v}_{j}^{r}} \cdot v_{j}^{r}+\frac{\partial P_{i j}}{\partial \hat{v}_{j}^{i}} \cdot v_{j}^{i} \\
& -g_{i j}^{n e t} \cdot\left[\left(\hat{v}_{i}^{r}\right)^{2}+\left(\hat{v_{i}^{i}}\right)^{2}\right]-G_{i j} \cdot\left(\hat{v_{i}^{r}} \cdot \hat{v}_{j}^{r}+\hat{v}_{i}^{i} \cdot \hat{v_{j}^{i}}\right) \\
& +B_{i j} \cdot\left(\hat{v_{i}^{r}} \cdot \hat{v}_{j}^{i}-\hat{v_{i}^{i}} \cdot \hat{v}_{j}^{r}\right), \quad \forall\{i, j\} \in 2 \mathcal{K} . \\
& Q_{i j}=\frac{\partial Q_{i j}}{\partial \hat{v}_{i}^{r}} \cdot v_{i}^{r}+\frac{\partial Q_{i j}}{\partial \hat{v_{i}^{i}}} \cdot v_{i}^{i}+\frac{\partial Q_{i j}}{\partial \hat{v}_{j}^{r}} \cdot v_{j}^{r}+\frac{\partial Q_{i j}}{\partial \hat{v}_{j}^{i}} \cdot v_{j}^{i} \\
& +b_{i j}^{n e t} \cdot\left[\left(\hat{v}_{i}^{r}\right)^{2}+\left(\hat{v_{i}^{i}}\right)^{2}\right]+B_{i j} \cdot\left(\hat{v}_{i}^{r} \cdot \hat{v}_{j}^{r}+\hat{v}_{i}^{i} \cdot \hat{v}_{j}^{i}\right) \\
& +G_{i j} \cdot\left(\hat{v_{i}^{r}} \cdot \hat{v_{j}^{i}}-\hat{v_{i}^{i}} \cdot \hat{v}_{j}^{r}\right), \forall\{i, j\} \in 2 \mathcal{K} . \\
& \sum_{g \in \mathcal{G}_{n}} p_{g}-\sum_{d \in \mathcal{D}_{n}} p_{d}=\sum_{k \in \mathcal{K}_{n}^{f}} P_{n k}+\sum_{k \in \mathcal{K}_{n}^{t}} P_{k n}+G_{n}^{L} \cdot \\
& \left.+2 \cdot \hat{v_{n}^{i}} \cdot v_{n}^{i}-\left(\hat{v}_{n}^{r}\right)^{2}-\left(\hat{v_{n}^{i}}\right)^{2}\right], \forall n \in \mathcal{N}
\end{aligned}
$$

$\sum_{g \in \mathcal{G}_{n}} q_{g}-\sum_{d \in \mathcal{D}_{n}} q_{d}=\sum_{k \in \mathcal{K}_{n}^{f}} Q_{n k}+\sum_{k \in \mathcal{K}_{n}^{t}} Q_{k n}-B_{n}^{L} \cdot[2$ $\left.+2 \cdot \hat{v_{n}^{i}} \cdot v_{n}^{i}-\left(\hat{v}_{n}^{r}\right)^{2}-\left(\hat{v_{n}^{i}}\right)^{2}\right], \forall n \in \mathcal{N}$

$2 \cdot \hat{v}_{n}^{r} \cdot v_{n}^{r}+2 \cdot \hat{v_{n}^{i}} \cdot v_{n}^{i}-\left(\hat{v}_{n}^{r}\right)^{2}-\left(\hat{v_{n}^{i}}\right)^{2} \geq\left(V_{n}^{M i n}\right)^{2}, \forall n \in \mathcal{N}$

$$
\begin{gathered}
\begin{aligned}
2 \cdot \hat{v}_{n}^{r} \cdot v_{n}^{r}+2 & \cdot \hat{v_{n}^{i}} \cdot v_{n}^{i}-\left(\hat{v_{n}^{r}}\right)^{2}-\left(\hat{v_{n}^{i}}\right)^{2} \leq\left(V_{n}^{M a x}\right)^{2}, \forall n \in \mathcal{N} . \\
2 \cdot \hat{P_{i j}} \cdot P_{i j}+ & 2 \cdot \hat{Q_{i j}} \cdot Q_{i j}-\hat{P_{i j}^{2}}-\hat{Q_{i j}^{2}} \leq\left(I_{i j}^{M a x}\right)^{2} \cdot\left[2 \cdot \hat{v_{n}^{r}} \cdot v_{n}^{r}\right. \\
& \left.+2 \cdot \hat{v_{n}^{i}} \cdot v_{n}^{i}-\left(\hat{v_{n}^{r}}\right)^{2}-\left(\hat{v_{n}^{i}}\right)^{2}\right], \forall\{i, j\} \in 2 \mathcal{K}
\end{aligned}
\end{gathered}
$$

$$
\begin{aligned}
& p_{g}^{\text {Min }} \leq p_{g} \leq p_{g}^{\text {Max }}, \forall g \in \mathcal{G} . \\
& q_{g}^{\text {Min }} \leq q_{g} \leq q_{g}^{\text {Max }}, \forall g \in \mathcal{G} .
\end{aligned}
$$

The main contribution of this paper is applying a hybrid of tuning methods and SLP to tackle the inherent challenges of a tractable ACOPF algorithm as well as the new challenges introduced by the linearized sub-problems. The linearized subproblems do not guarantee convergence on their own. Solving only a set of linearized sub-problems can result in diverging the nonlinear ACOPF problem from a feasible solution. The tuning methods we propose are able to effectively address the above challenges and significantly reduce the number of iterations required to solve an ACOPF problem. The proposed tuning methods are:

1. Addition of a random noise to the generator cost functions in order to eliminate degeneracy with respect to active power decisions.

2. Enforcing convergence constraints.

3. Adding a wrap-around constraint for real power balance.

4. Enforcing constraints that penalize deviation from unity voltage.

5. Replacing some non-convex constraints with penalties in the objective function.

6. Splitting the iteration process into three stages to further take advantage of SLP's sensitivity to initialization, in addition to initializing the algorithm with DCOPF solution. This technique ensures that a poor solution does not directly initialize the fully-constrained ACOPF problem.

With the help of these tuning methods, our approach first searches for an optimal solution to a relatively relaxed ACOPF problem (i.e., during Stage 1). A feasible solution (i.e., during Stage 2) to the constrained ACOPF problem is sought in the proximity of the optimal solution found at Stage 1 .

\section{The Proposed Algorithm}

The algorithm that we implemented in this paper is described $v_{n}^{r}$ by the flowchart shown in fig. 2. As mentioned in previous sections, the idea is based on exploiting the sensitivity of the SLP method to the initialization. Thus, each stage acts as initialization to the next.

The algorithm starts by adding a small random noise to the $v_{n}^{r}$ real power generation cost to eliminate degeneracy with respect to active power decisions. Additionally, the performance of successive linear programming in solving nonlinear optimization problems is substantially impacted by initialization. Thus, to improve the algorithm's performance, we included a warm-start initialization. We initialize the SLP algorithm with a DCOPF solution. The SLP algorithm comprises three stages 


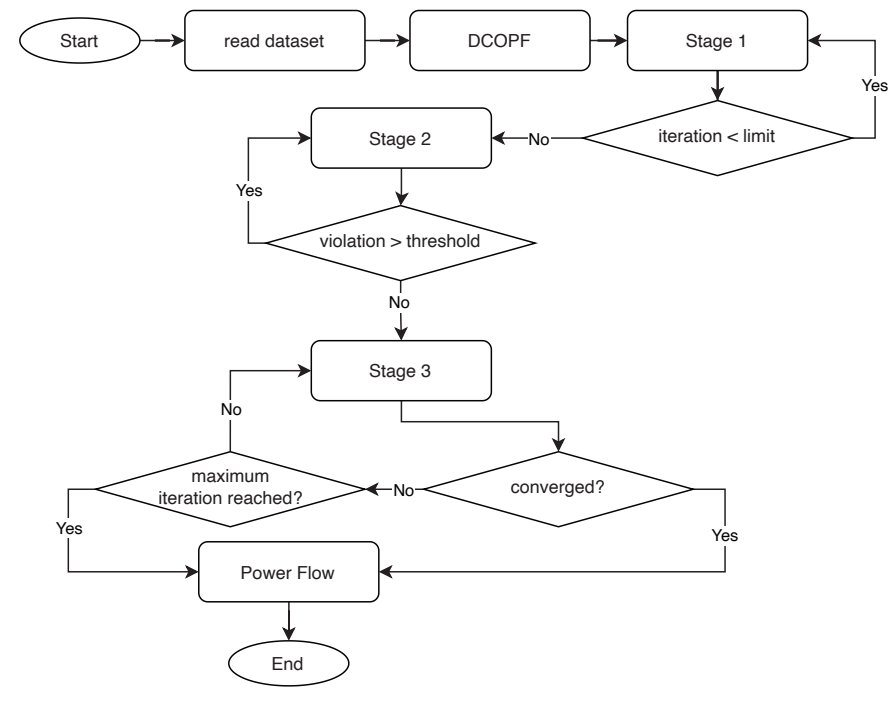

Figure 2: Flowchart of our proposed algorithm.

followed by a power flow calculation to ensure a feasible solution at the end of the algorithm. Stage 1 is initialized by a DCOPF solution and consists of an SLP formulation of the ACOPF problem with relaxed voltages and line thermal limits. This stage serves as a partially unbounded AC initialization for the next step (i.e., Stage 2, which is an SLP sub-problem with voltage and branch limits). To keep the voltages close to their bounds in all three stages, we enforce a penalty on bus voltage deviations from unity. Results with trial networks demonstrate that the addition of these penalties leads to satisfactory performance and keeps the voltages close to their limits. A similar approach is adopted to address the non-convexity of lower bounds of voltages in Stage 2 and Stage 3, where we do not directly enforce the voltage lower bound constraints to avoid trimming any part of the feasible region from the linearized solution space.

In Stage 2, we add constraints to enforce line thermal limits and upper bounds of the nodal voltages. This stage aims to find an optimal solution to the ACOPF problem with voltage and line limits. This stage solves any violation resulting from the enforcement of line and voltage limits. In Stage 3, we add three more forces that penalize any real and reactive power dispatch changes. These penalties contribute to a rapid convergence in the algorithm. In addition, our experiments have shown a contribution to the overall performance by the scalar wrap-around constraint, which ensures the total real power generated is always equal or greater than the total real power load.

We implement the power flow algorithm using Argonne National Lab's Portable, Extensible Toolkit for Scientific Computation (PETSc). Overall, the results of our numerical experiments on GO competition's Challenge 1 trial networks show satisfactory performance and scalability of the SLP algorithm proposed in this paper.

\subsection{DCOPF Initialization}

As stated before, any solution better than a random or flat cold start can surely speed up the SLP algorithm. Therefore, we initialize our algorithm with DCOPF solution, which is an LP problem shown in (6). A DCOPF is a linear programming (LP) model which is readily available in the existing energy management systems (EMS) and therefore, an appropriate candidate for the initialization of our proposed algorithm in the industry implementation. A DCOPF model approximates the ACOPF where voltage magnitudes are assumed to be 1 p.u. across all buses. It also assumes the network to be lossless and considers the transmission branches' reactive power flow to be zero.

$$
\begin{array}{ll}
\operatorname{minimize} & \sum_{g \in \mathcal{G}} c_{g} \\
\text { subject to } & c_{g}=\sum_{h \in \mathcal{H}_{g}} c_{g h} \cdot t_{g h}, \forall g \in \mathcal{G} . \\
& p_{g}=\sum_{h \in \mathcal{H}_{g}} p_{g h} \cdot t_{g h}, \forall g \in \mathcal{G} . \\
& \sum_{h \in \mathcal{H}_{g}} t_{g h}=1, \forall g \in \mathcal{G} . \\
& t_{g h} \geq 0, \forall g \in \mathcal{G}, h \in \mathcal{H}_{g} \\
& P_{i j}=g_{i j}^{\text {net }}+G_{i j}+B_{i j} \cdot\left(\theta_{i}-\theta_{j}\right), \forall\{i, j\} \in 2 \mathcal{K} \\
& \sum_{g \in \mathcal{G}_{n}} p_{g}-\sum_{d \in \mathcal{D}_{n}} p_{d}=\sum_{k \in \mathcal{K}_{n}^{f}} P_{n k}+\sum_{k \in \mathcal{K}_{n}^{t}} P_{k n}+G_{n}^{L}, \forall n \in \mathcal{N} . \\
& p_{g}^{\text {Min }} \leq p_{g} \leq p_{g}^{\text {Max }}, \forall g \in \mathcal{G}
\end{array}
$$

where $v_{n}^{r}=\cos \left(\theta_{n}\right)$ and $v_{n}^{i}=\sin \left(\theta_{n}\right) \forall n \in \mathcal{N}$.

\subsection{Stage 1}

In this stage, we solve (7), which has all the constraints from (5) except (5j), (5k) and (51), which relaxes the voltage and line thermal limits. The additional constraints $(7 \mathrm{~d})$ is added so that while voltage constraints are relaxed, deviation from unity voltage is penalized by a small value in the objective function $7 \mathrm{7a}$. These additional constraints help the majority of the voltage to remain within their limits. We also add a wrap-around constraint shown in (7c). Our experiments show that although this constraint may seem duplicate, it provides a tighter trust-region for the real power in the LP subproblem and therefore, helps in the algorithm's speedy convergence. We initialize (7) with the solution of DCOPF shown in (6).

$$
\begin{array}{ll}
\operatorname{minimize} & \sum_{g \in \mathcal{G}} c_{g}+W_{v} \sum_{n \in \mathcal{N}}\left(a_{v n}+b_{v n}\right) \\
\text { subject to } & \\
& \sum_{g \in \mathcal{G}} p_{g} \geq \sum_{d \in \mathcal{D}} p_{d} \\
& 2\left(\hat{v}_{n}^{1 r} v_{n}^{r}+\hat{v}_{n}^{1 i} v_{n}^{i}\right)-\left(\hat{v}_{n}^{1 r}\right)^{2}-\left(\hat{v}_{n}^{1 i}\right)^{2}=a_{v n}-b_{v n}, \forall n \in \mathcal{N} .
\end{array}
$$

\subsection{Stage 2}

In this stage, we solve (8), which has all the constraints from (7), in addition to voltage upper bounds in $8 \mathrm{c}$ and transmission branch thermal limits in $8 \mathrm{e}$. The voltage lower bound 
constraints are non-convex constraints and a direct imposition of its linearized equivalence can possibly trim some of the feasible space from the ACOPF problem in the LP sub-problem. Therefore, instead of directly imposing lower bound voltage constraints, we penalize any violations of the voltage lower bounds as shown in $8 \mathrm{~d}$. Constraints in $8 \mathrm{c}-8 \mathrm{e}$ are only enforced on violating variables based on estimations from preceding iteration using constraints reduction approach.

Additional box constraints are added to some variables such as nodal voltages and branch power flows as defined by $8 \mathrm{8f}$ 8ii. An illustrative example of a box constraint is shown in Fig. 3 for a nodal voltage at bus $n$.

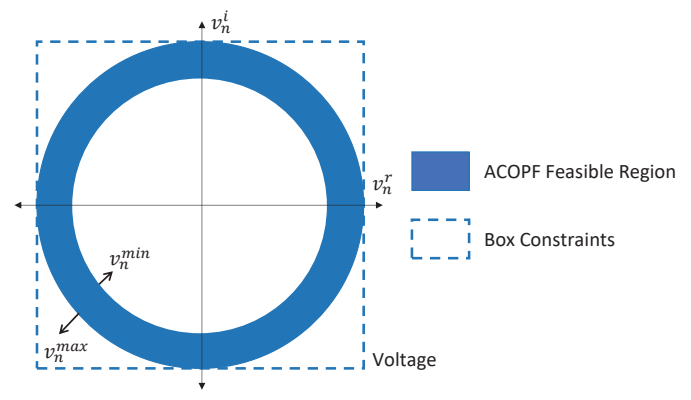

Figure 3: Illustration of a box constraint for the nodal voltage at bus $n$ [13].

Box constraints are a practical approach for alleviating the nonlinear constraints in optimization problems. They help in the convergence of first order nonlinear programming methods such as the SLP method where the linearizations may not be necessarily bounded, and therefore trust region techniques such as box constraints are needed to ensure convergence in theory. We can also add contingency constraints in this stage if we are solving an SC-ACOPF problem (8f) - (8i).

$\operatorname{minimize} \sum_{g \in \mathcal{G}} c_{g}+W_{v} \sum_{n \in \mathcal{N}}\left(a_{v n}+b_{v n}\right)+W_{v M i n} \sum_{n \in \mathcal{N}}\left(a_{v M i n_{n}}+b_{v \operatorname{Min}_{n}}\right)$

subject to $7 \mathrm{~b}-(7 \mathrm{~d})$

\subsection{Stage 3}

In this stage, we solve (9), which has all the constraints from (8), in addition to the convergence constraints in 9c) - (9e). These new constraints help in pushing the algorithm for convergence. This stage may slightly compromise the optimality of the problem. However, the advantage of the convergence constraints in attaining faster convergence far exceeds the minor optimality compromise.

$$
\begin{aligned}
& \operatorname{minimize}+W_{p g} \sum_{g \in \mathcal{G}}\left(a_{p g}+b_{p g}\right) \\
&+W_{q g} \sum_{g \in \mathcal{G}}\left(a_{q g}+b_{q g}\right) \\
&+W_{B c s} \sum_{n \in \mathcal{N}}\left(a_{B c s_{n}}+b_{B c s_{n}}\right)
\end{aligned}
$$

subject to $8 \mathrm{~b}-8 \mathrm{i}$

$$
\begin{aligned}
& p_{g}-\hat{p}_{g}=a_{p g}-b_{p g}, \quad \forall g \in \mathcal{G} . \\
& q_{g}-\hat{q}_{g}=a_{q g}-b_{q g}, \forall g \in \mathcal{G} . \\
& b_{s}^{c s}-b_{s}^{\hat{c} s}=a_{B c s}-b_{B c s}, \quad \forall n \in \mathcal{N} .
\end{aligned}
$$

\subsection{Power Flow}

Solving power flow initialized by the solution from Stage 3 is the last step in our algorithm. In most cases, the power flow serves as an accuracy enhancement stage and a final feasibility verification step. However, for the cases where the maximum number of iterations is reached in Stage 3, it also serves as a last resort to find a feasible solution for the problem. Many tools implement power flow, and most of them are quite fast. Our algorithm implements the Newton-Raphson method using Argonne National Laboratory's PETSc tool as shown in (10). The tool solves the system of nonlinear equations. Since it is initialized by a solution feasible or close to feasibility, it is solved in almost all test cases in less than 2 iterations.

$$
\left[\begin{array}{l}
\theta \\
V
\end{array}\right]^{k+1}=\left[\begin{array}{l}
\theta \\
V
\end{array}\right]^{k}+\mathcal{J}^{-1}\left(\left[\begin{array}{l}
\theta \\
V
\end{array}\right]^{k}\right)\left[\begin{array}{l}
f_{P}(\theta, V) \\
f_{Q}(\theta, V)
\end{array}\right]^{k}
$$

where $\mathcal{J}$ is a Jacobian Matrix. $f_{P}(\theta, V)$ and $f_{Q}(\theta, V)$ are power mismatches and are calculated as,

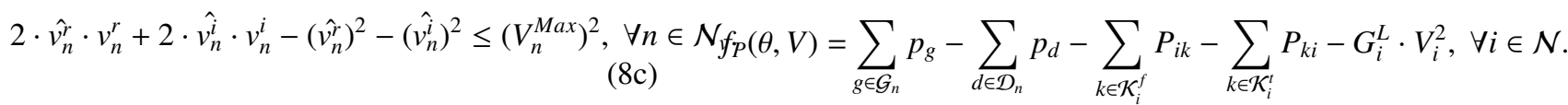

$$
\begin{aligned}
& 2 \cdot \hat{v}_{n}^{\operatorname{Min}^{r}} \cdot v_{n}^{r}+2 \cdot \hat{v}_{n}^{M i n^{i}} \cdot v_{n}^{i}-\left(\hat{v}_{n}^{\operatorname{Min}^{r}}\right)^{2}-\left(\hat{v}_{n}^{\text {Min }^{i}}\right)^{2}=a_{v M i n_{n}} \\
& -b_{v \operatorname{Min}_{n}}, \forall n \in \mathcal{N}_{v} \text {. } \\
& f_{Q}(\theta, V)=\sum_{g \in \mathcal{G}_{n}} q_{g}-\sum_{d \in \mathcal{D}_{n}} q_{d}-\sum_{k \in \mathcal{K}_{i}^{f}} Q_{i k}-\sum_{k \in \mathcal{K}_{i}^{t}} Q_{k i}+B_{i}^{L} \cdot V_{i}^{2}, \forall i \in \mathcal{N} .
\end{aligned}
$$

$$
\begin{aligned}
2 \cdot \hat{P}_{i j} \cdot P_{i j}+ & 2 \cdot \hat{Q}_{i j} \cdot Q_{i j}-P_{i j}^{2}-Q_{i j}^{2} \leq\left(I_{i j}^{M a x}\right)^{2} \cdot\left(2 \cdot \hat{v_{n}^{r}} \cdot v_{n}^{r}\right. \\
& \left.+2 \cdot v_{n}^{i} \cdot v_{n}^{i}-\left(\hat{v_{n}^{r}}\right)^{2}-\left(\hat{v}_{n}^{i}\right)^{2}\right), \forall\{i, j\} \in 2 \mathcal{K}_{v} .
\end{aligned}
$$

$-V_{n}^{\operatorname{Max}} \leq v_{n}^{r} \leq V_{n}^{\operatorname{Max}}, \forall n \in \mathcal{N}$.

$-V_{n}^{\operatorname{Max}} \leq v_{n}^{i} \leq V_{n}^{\operatorname{Max}}, \forall n \in \mathcal{N}$.

$-I_{i j}^{\text {Max }} \cdot V_{n}^{\text {Max }} \leq P_{i j} \leq I_{i j}^{\text {Max }} \cdot V_{n}^{\text {Max }}, \forall\{i, j\} \in 2 \mathcal{K}$

$-I_{i j}^{M a x} \cdot V_{n}^{M a x} \leq Q_{i j} \leq I_{i j}^{M a x} \cdot V_{n}^{\text {Max }}, \forall\{i, j\} \in 2 \mathcal{K}$

\section{Numerical Experiments}

Various numerical experiments were performed on a 4-core Intel(R) i7-7700 CPU @ 3.60GHz 8GB RAM system using Julia language v1.1.0, JuMP v0.18 for mathematical optimization modelling, and Gurobi v9.0.0 as an linear programming solver, to test and validate the performance of the proposed algorithm. Table 1 describes the test networks and the results of our numerical experiments. In table 1 , we also introduce optimality 
performance metrics $\eta$ to measure the betterment or worsening of optimality of the proposed SLP method's solution compared to the IPOPT solver. $\eta$ is defined in (12).

$$
\eta=\frac{O b j_{S L P}-O b j_{I P O P T}}{O b j_{\perp P O P T}} \times 100
$$

Table 1: The table describes the test networks and the results of our numerical experiments.

\begin{tabular}{|c|c|c|c|c|c|c|c|c|}
\hline \hline \multirow{2}{*}{ Networks } & \multirow{2}{*}{ Buses } & \multirow{2}{*}{ Gens } & \multirow{2}{*}{ Branches } & \multicolumn{2}{|c|}{ IPOPT } & \multicolumn{2}{|c|}{ SLP } & \multirow{2}{*}{$\eta$} \\
\cline { 5 - 9 } & & & & iter & runtime (s) & iter & runtime (s) & \\
\hline Network_02R074 & 500 & 224 & 733 & 51 & 32.24 & 7 & 23.59 & 0.02 \\
\hline Network_03R200 & 793 & 214 & 913 & 54 & 34.55 & 12 & 28.41 & 1.35 \\
\hline Network_06R113 & 2000 & 384 & 3639 & 56 & 62.44 & 12 & 43.87 & 0.32 \\
\hline Network_70R422 & 2312 & 444 & 3013 & 60 & 63.31 & 12 & 48.36 & 3.38 \\
\hline Network_76R080 & 2755 & 170 & 4763 & 125 & 167.1 & 12 & 49.92 & 0.87 \\
\hline Network_83R130 & 4020 & 345 & 6988 & 408 & 936.78 & 9 & 59.82 & 2.15 \\
\hline Network_86R050 & 4619 & 348 & 8150 & 60 & 132.82 & 15 & 98 & 1.95 \\
\hline Network_09R064 & 4918 & 1340 & 6727 & 94 & 141.44 & 16 & 223.8 & 9.08 \\
\hline Network_09R064 & 8733 & 743 & 14269 & 89 & 308.76 & 13 & 257.2 & 8.05 \\
\hline Network_13R015 & 10000 & 2089 & 13193 & 102 & 292.07 & 11 & 255.8 & 8.38 \\
\hline Network_13R088 & 10000 & 2089 & 13193 & 79 & 226.23 & 12 & 280.43 & 3.56 \\
\hline Network_15R010 & 11612 & 899 & 19903 & 747 & 2502.37 & 15 & 1450.23 & 8.18 \\
\hline Network_15R030 & 11615 & 868 & 19902 & 794 & 4366.25 & 12 & 1181.97 & 9.09 \\
\hline Network_20R100 & 19402 & 973 & 34698 & 93 & 561.99 & 11 & 1746.58 & 9.91 \\
\hline Network_30R044 & 30000 & 3526 & 35393 & 446 & 3294.52 & 15 & 2536.11 & 9.17 \\
\hline \hline
\end{tabular}

The results are also plotted in fig. 4,5, 6, and 7, By observing the plots, we can see a few important and some unique characteristics of our proposed algorithms which are described below and has been validated over a spectrum of test cases ranging from 500-bus systems to 30,000-bus systems. The test cases used in our numerical experiments are from the datasets of the U.S. Department of Energy's Grid Optimization Competition Challenge 1 [44].

\section{Computation time:}

The plot in fig. 4 shows the computation time for different test networks using the proposed algorithm. It also compares the computation time of the proposed algorithm with the IPOPT's computation time for solving the same test cases. The plot shows that more than $\% 80$ of the test networks were solved faster by the proposed method than the IPOPT solver. This is especially significant because IPOPT implements the Nonlinear Interior-Point Line Search Filter method, which is a second-order method, and our proposed algorithm is a firstorder method. We observed that in the cases where IPOPT performs better, the DCOPF provides a poor initialization for Stage 1 in our proposed algorithm. Particularly, the reactive power balance violation is higher in these cases than the remaining cases. In other words, these cases have high reactive power flow which is assumed zero in DCOPF. Ideally, second-order methods are expected to solve faster than first-order methods since the sub-problems in the second-order methods carry some of the curvature information, leading to faster convergence. Thus, the plot shows that the heuristics applied by tuning methods in the proposed algorithm has significantly improved the performance (i.e., computation time) of the SLP method. The total time for solving all the test cases by our proposed algorithm is $36.8 \%$ faster than IPOPT.

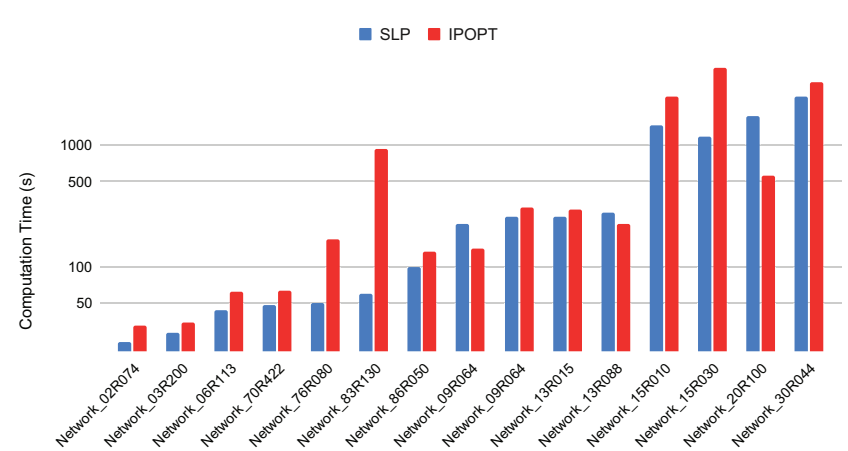

Figure 4: The plot compares the computation time of the proposed algorithm with the IPOPT solver. For better visual of the results, the vertical axis of the plot is log-scaled.

\section{Number of iterations:}

The plot in fig. 5 shows the number of iterations the proposed algorithm need to solve different test networks. It shows a significant reduction in the total number of iterations needed to solve the problem compared to IPOPT. This again verifies the comparable performance of the proposed algorithm with a second-order method. Although, the sub-problems of the proposed algorithm may not have the same complexity as the subproblems solved in IPOPT solver, the significant reduction in the number of iterations needed to solve an ACOPF problem validates the merit of the proposed algorithm.

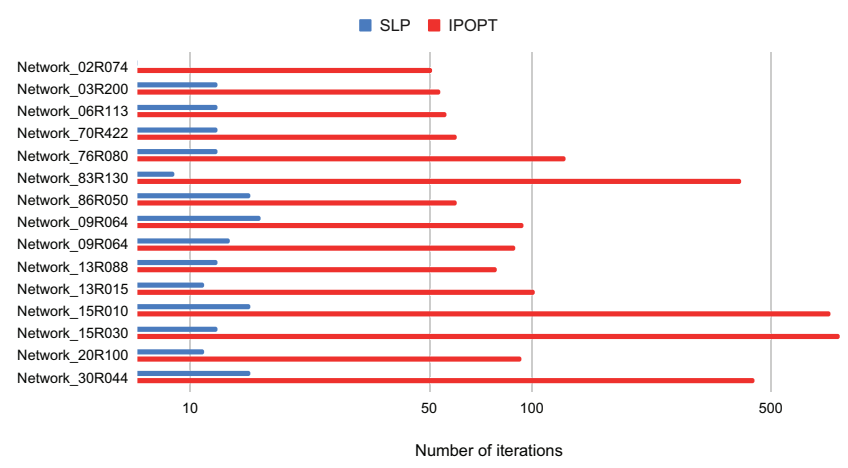

Figure 5: The plot compares the number of iterations needed to solve the ACOPF problem for different test networks using the proposed algorithm to the number of iterations in the IPOPT solver. For better visual of the results, the vertical axis of the plot is log-scaled.

\section{Scalability:}

The plots in fig 6 and 7 show the correlation between performance indicating parameters (i.e., computation time and number of iterations) and the size of the ACOPF problem (i.e., number of buses). Fig. 6 shows linear scalability of our algorithm to the size of the problem. The linear scalability has a high coefficient of determination (i.e., $R^{2}=0.931$ ).

On the other hand, there is no significant correlation between the size of the problem and the number of iterations needed to 
solve the problem by the proposed algorithm as shown in fig. 7 The same cannot be said about the IPOPT solver as can be seen in fig. 5. In other words, the number of iterations needed to solve a problem by the proposed algorithm remains almost the same for all sizes of the problem (i.e., $R^{2}=0.085$ ). This also means that the increase in the computation time corresponding to the increase in the problem's size is almost entirely caused by the increase in the LP sub-problems' complexity.

This is another important property of the proposed algorithm, which points to its high potential for the application of highperformance computation (HPC). The proposed algorithm can be easily further sped up by either reducing the complexity of the LP sub-problems of the proposed algorithm, applying parallel and high-performance computation techniques to the proposed algorithm's sub-problems, or both.

Finally, to make our results reproducible, we chose consistent coefficients of penalties in the objective function associated with tuning methods for different networks, which has resulted in relatively high optimality gap shown by $\eta$ in some of the test networks in table 1] The proposed method can yield lower optimality gaps (i.e., $\eta$ value in table 1 if these coefficients are optimized for each network. For future work, the proposed algorithm can be extended to incorporate other devices such as FACTS devices, Transformer Tap settings, Phase Shifting Transformers (PST), etc.

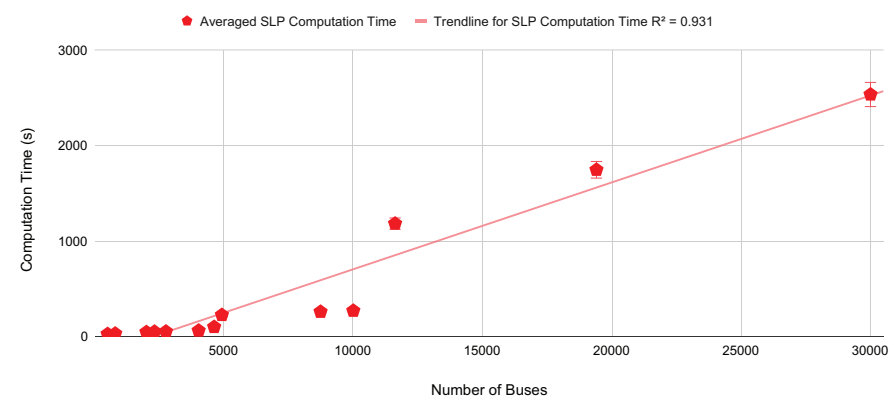

Figure 6: The plots give the coefficient of determination for the correlation between performance indicators (i.e., computation time) and the size of the ACOPF problem in the proposed algorithm.

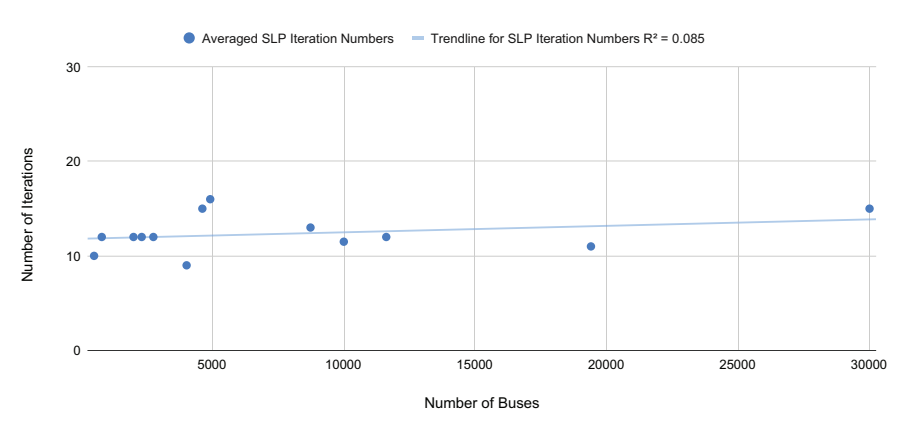

Figure 7: The plots give the coefficient of determination for the correlation between performance indicators (i.e., number iterations) and the size of the ACOPF problem in the proposed algorithm.

\section{Conclusion}

This paper presents a heuristic algorithm for solving the ACOPF problem. The proposed method combines various tuning methods and a sequential linear programming approach. The heuristic approach is mainly proposed for large-scale problems and tackles the computational challenges associated with it. Numerical experiments show that the proposed algorithm, tested on the Grid Optimization Competition's Challenge I test cases, solves ACOPF problems in significantly fewer iterations than the IPOPT solver. Although the proposed algorithm is a first-order approximation method, it can solve more than $80 \%$ of the test networks faster than the IPOPT solver, which implements a nonlinear interior-point algorithm, a second-order approximation method. At a slight compromise on the optimality, the proposed method solves large-scale problems within the industry time-constraints.

\section{Appendix A. Nomenclature}

Table A.2 describes the convention for primitive index sets used throughout the paper. Table A.3 describes the convention for data parameters used throughout the paper. Table A.4 describes the convention for optimization variables used throughout the paper.

Table A.2: Primitive Index Sets

\begin{tabular}{|c|l|}
\hline \hline Symbol & Description \\
\hline \hline $\mathcal{G}$ & Set of generators \\
\hline $\mathcal{D}$ & Set of loads \\
\hline $\mathcal{D}_{n}$ & Set of loads connected to bus $n$ \\
\hline $\mathcal{K}$ & Set of lines (Line branches and transformers branches) \\
\hline $\mathcal{K}_{v}$ & Subset of lines $\mathcal{K}$ that has violation of a variable or a parameter \\
\hline $\mathcal{K}_{n}^{f}$ & Set of "from" (i.e. $i$ in $\{i, j\}$ ) lines $\in \mathcal{K}$ connected to bus $\mathrm{n}$ \\
\hline $\mathcal{K}_{n}^{t}$ & Set of "to" (i.e. $j$ in $\{i, j\}$ ) lines $\in \mathcal{K}$ connected to bus $\mathrm{n}$ \\
\hline $\mathcal{N}$ & Set of buses \\
\hline $\mathcal{N}_{v}$ & Subset of of buses $\mathcal{N}$ that has violation of a variable or a parameter \\
\hline $\mathcal{H}_{g}$ & Set of cost function sample points for generator $g$ \\
\hline \hline
\end{tabular}

Table A.3: Data Parameters

\begin{tabular}{|c|c|}
\hline Symbol & Description \\
\hline$c_{2 g}, c_{1 g}, c_{0 g}$ & Coefficients of quadratic cost function of generator $g$ \\
\hline$\tau_{i j}$ & Complex transformer ratio of line $\{i, j\}$ \\
\hline$Y_{i j}$ & Complex (i.e., $G_{i j}+j B_{i j}$ ) admittance matrix element for node $i$ and $j$ \\
\hline$y_{i j}$ & Complex (i.e., $g_{i j}+j b_{i j}$ ) series impedance between node $i$ and $j$ \\
\hline$y_{i j}^{s}$ & Complex (i.e., $g_{i j}^{s}+j b_{i j}^{s}$ ) charging impedance between node $i$ and $j$ \\
\hline$\frac{y_{i j}}{y_{i j}^{M}}$ & Complex (i.e., $g_{i j}^{M}+j b_{i j}^{M}$ ) magnetizing impedance between node $i$ and $j$ \\
\hline$s_{d}$ & Complex load (i.e., $\left.p_{d}+j q_{d}\right)$ \\
\hline$Y_{n}^{L}$ & Complex Shunt Element (i.e., $\left.G_{n}^{L}-j B_{n}^{L}\right)$ connected to bus $n$ \\
\hline$V_{n}^{\text {Min }}, V_{n}^{\text {Max }}$ & Lower and upper voltage bounds at bus $n$ \\
\hline$I_{i j}^{\operatorname{Max}}$ & Line thermal limit of line $\{i, j\}$ \\
\hline$s_{g}^{\text {Min }}, s_{g}^{\text {Max }}$ & Complex (i.e., $p_{g}^{\operatorname{Min} / \operatorname{Max}}+j q_{g}^{\operatorname{Min} / \operatorname{Max}}$ ) generator $g$ bounds \\
\hline$p_{g h}$ & Real power output of generator $g$ at sample point $h$ (p.u.) \\
\hline$c_{g h}$ & Generation cost of generator $g$ at sample point $h(\mathrm{USD} / \mathrm{h})$ \\
\hline$\hat{v}_{n}^{H}$ & Complex (i.e., $\hat{v}_{n}^{H r}+j \hat{v}_{n}^{H i}$ ) history estimated voltage at bus $n$ \\
\hline$\hat{v}_{n}^{1}=\hat{v}_{n} /\left|\hat{v}_{n}\right|$ & Complex (i.e., $\hat{v}_{n}^{1 r}+j \hat{v}_{n}^{I i}$ ) estimated unity voltage at bus $n$. \\
\hline$\hat{v}_{n}^{\operatorname{Min}}=\hat{v}_{n} \cdot V_{n}^{\operatorname{Min}} /\left|\hat{v}_{n}\right|$ & Complex (i.e., $\hat{v}_{n}^{\text {Min' }}+j \hat{v}_{n}^{\text {Min }}$ ) ) estimated minimum voltage at bus $n$ \\
\hline$W_{p g} / W_{q g}$ & Penalty for deviation of $p_{g} / q_{g}$ from estimated $\hat{p}_{g} / \hat{q}_{g}$ \\
\hline$W_{v} / W_{v \text { Min }}$ & Penalty for deviation of $v_{n}$ from estimated $\hat{v}_{n}^{1}$ or violating $V_{n}^{M i n}$ \\
\hline
\end{tabular}


Table A.4: Optimization Variables

\begin{tabular}{|c|l|}
\hline \hline Symbol & Description \\
\hline \hline$c_{g}$ & Generation cost of generator $g$ (USD/h) \\
\hline$s_{g}$ & Generator $g$ apparent power output (p.u.) \\
\hline$p_{g}$ & $p_{g}=\mathfrak{R}\left[s_{g}\right]$ real part of $s_{g}$ representing the real power output of generator $g$ in (p.u.) \\
\hline$q_{g}$ & $q_{g}=\mathfrak{I}\left[s_{g}\right]$ imaginary part of $s_{g}$ representing the reactive power output of gen $g$ (p.u.) \\
\hline$v_{n}$ & Complex (i.e., $v_{n}^{r}+j v_{n}^{i}$ ) voltage at bus $n$ (p.u.) \\
\hline$S_{i j}$ & Complex (i.e., $P_{i j}+j Q_{i j}$ ) line apparent power flow from node $i$ to $j$ (p.u.) \\
\hline$S_{n}^{g}$ & Complex (i.e., $P_{n}^{g}+j Q_{n}^{g}$ ) nodal net generative injection at node $n$ (p.u.) \\
\hline$t_{g h}$ & Coefficient of point $h$ for generator $g$ solution as a point on generation cost function \\
\hline$a_{p g}$ & Variable to catch the positive component of deviation of $p_{g}$ from $\hat{p}_{g}$ for generator $g$. \\
\hline$b_{p g}$ & Variable to catch the negative component of deviation of $p_{g}$ from $\hat{p}_{g}$ for generator $g$. \\
\hline$a_{q g}$ & Variable to catch the positive component of deviation of $q_{g}$ from $\hat{q}_{g}$ for generator $g$. \\
\hline$b_{q g}$ & Variable to catch the negative component of deviation of $q_{g}$ from $\hat{q}_{g}$ for generator $g$. \\
\hline$a_{v M i_{n}}$ & Variable to catch the positive component of deviation of $v_{n}$ from $\hat{v}_{n}^{M i n}$ at node $n$. \\
\hline$b_{v M i_{n}}$ & Variable to catch the negative component of deviation of $v_{n}$ from $\hat{v}_{n}^{\text {Min }}$ at node $n$. \\
\hline$a_{v n}$ & Variable to catch the positive component of deviation of $v_{n}$ from $\hat{v}_{n}^{1}$ at node $n$. \\
\hline$b_{v n}$ & Variable to catch the negative component of deviation of $v_{n}$ from $\hat{v}_{n}^{1}$ at node $n$. \\
\hline \hline
\end{tabular}

\section{References}

[1] M. B. Cain, R. P. O'neill, A. Castillo, et al., History of optimal power flow and formulations, Federal Energy Regulatory Commission 1 (2012) $1-36$.

[2] S. A. Sadat, D. Haralson, M. Sahraei-Ardakani, Security versus computation time in iv-acopf with socp initialization, in: 2018 IEEE International Conference on Probabilistic Methods Applied to Power Systems (PMAPS), 2018, pp. 1-6. doi:10.1109/PMAPS .2018.8440287

[3] A. Castillo, R. P. O'Neill, Computational performance of solution techniques applied to the acopf, Federal Energy Regulatory Commission, Optimal Power Flow Paper 5 (2013).

[4] Energy Information Administration, Revenue from sales of electricity to ultimate customers U.S. Federal Statistical System (2019). URL https://www.eia.gov/electricity/annual/html/epa_ 02_03.html

[5] B. Stott, J. Jardim, O. Alsaç, Dc power flow revisited, IEEE Transactions on Power Systems 24 (3) (2009) 1290-1300.

[6] S. A. Sadat, L. Fan, Mixed integer linear programming formulation for chance constrained mathematical programs with equilibrium constraints, 2017 IEEE Power and Energy Society General Meeting (PESGM) (2017) $1-5$.

[7] B. Singh, R. Mahanty, S. Singh, Centralized and decentralized optimal decision support for congestion management, International Journal of Electrical Power \& Energy Systems 64 (2015) 250-259. doi:https : //doi.org/10.1016/j.ijepes.2014.07.026

[8] A. Castillo, P. Lipka, J.-P. Watson, S. S. Oren, R. P. O’Neill, A successive linear programming approach to solving the IV-ACOPF, IEEE Transactions on Power Systems 31 (4) (2015) 2752-2763.

[9] K. Hedman, S. Oren, R. O’Neill, Optimal transmission switching: Economic efficiency and market implications, Journal of Regulatory Economics 40 (2) (2011) 111-140. doi:10.1007/s11149-011-9158-z

[10] A. Chaib, H. Bouchekara, R. Mehasni, M. Abido, Optimal power flow with emission and non-smooth cost functions using backtracking search optimization algorithm International Journal of Electrical Power \& Energy Systems 81 (2016) 64-77. doi:https://doi.org/10.1016/j. ijepes.2016.02.004

URL https://www.sciencedirect.com/science/article/pii/ S0142061516000788

[11] T. Summers, J. Warrington, M. Morari, J. Lygeros, Stochastic optimal power flow based on conditional value at risk and distributional robustness, International Journal of Electrical Power \& Energy Systems 72 (2015) 116-125, the Special Issue for 18th Power Systems Computation Conference. doi:https://doi.org/10.1016/j.ijepes.2015.02. 024

[12] J. Nocedal, S. Wright, Numerical optimization. 2nd edn springer, New York (2006).

[13] S. A. Sadat, K. Kim, Numerical performance of different formulations for alternating current optimal power flow, 2021 Australasian Universities Power Engineering Conference (AUPEC) (2021) 1-6.

[14] J. Kardos, D. Kourounis, O. Schenk, R. Zimmerman, Complete results for a numerical evaluation of interior point solvers for large-scale optimal power flow problems (2020). arXiv:1807.03964

[15] M. Lu, Optimization Methods in Electric Power Systems: Global Solutions for Optimal Power Flow and Algorithms for Resilient Design under Geomagnetic Disturbances, Ph.D. thesis, Clemson University (2018).

[16] S. H. Low, Convex relaxation of optimal power flow-part i: Formulations and equivalence, IEEE Transactions on Control of Network Systems 1 (1) (2014) 15-27.

[17] H. Hijazi, C. Coffrin, P. Van Hentenryck, Convex quadratic relaxations for mixed-integer nonlinear programs in power systems, Mathematical Programming Computation 9 (3) (2017) 321-367.

[18] J. Lavaei, S. H. Low, Zero duality gap in optimal power flow problem, IEEE Transactions on Power Systems 27 (1) (2012) 92-107.

[19] R. Madani, S. Sojoudi, J. Lavaei, Convex relaxation for optimal power flow problem: Mesh networks, IEEE Transactions on Power Systems 30 (1) (2015) 199-211.

[20] X. Bai, H. Wei, K. Fujisawa, Y. Wang, Semidefinite programming for optimal power flow problems International Journal of Electrical Power \& Energy Systems 30 (6) (2008) 383-392. doi:https://doi.org/10. $1016 /$ j.ijepes.2007.12.003

URL https://www.sciencedirect.com/science/article/pii/ S0142061507001378

[21] S. Bose, S. H. Low, T. Teeraratkul, B. Hassibi, Equivalent relaxations of optimal power flow, IEEE Transactions on Automatic Control 60 (3) (2015) 729-742. doi:10.1109/TAC.2014.2357112

[22] S. H. Low, Convex relaxation of optimal power flow-part ii: Exactness, IEEE Transactions on Control of Network Systems 1 (2) (2014) 177-189. doi:10.1109/TCNS.2014.2323634

[23] A.-F. Attia, R. A. El Sehiemy, H. M. Hasanien, Optimal power flow solution in power systems using a novel sine-cosine algorithm International Journal of Electrical Power \& Energy Systems 99 (2018) 331-343. doi:https://doi.org/10.1016/j.ijepes.2018.01.024 URL https://www.sciencedirect.com/science/article/pii/ S0142061517330272

[24] G. L. Torres, V. H. Quintana, An interior-point method for nonlinear optimal power flow using voltage rectangular coordinates, IEEE Transactions on Power Systems 13 (4) (1998) 1211-1218. doi:10.1109/59. 736231

[25] G. L. Torres, M. A. De Carvalho, On efficient implementation of interiorpoint based optimal power flows in rectangular coordinates, in: 2006 IEEE PES Power Systems Conference and Exposition, 2006, pp. 17471752. doi:10.1109/PSCE. 2006.296177

[26] E. Wong, Active-set methods for quadratic programming, Ph.D. thesis, University of California San Diego (2011).

[27] R. P. O'Neill, A. Castillo, M. B. Cain, The IV formulation and linear approximations of the ac optimal power flow problem, Published online at http://www. ferc. gov/industries/electric/indus-act/market-planning/opfpapers/acopf-2-iv-linearization. pdf (2012).

[28] R. Byrd, N. Gould, J. Nocedal, R. A. Waltz, An algorithm for nonlinear optimization using linear programming and equality constrained subproblems, Mathematical Programming 100 (2004) 27-48.

[29] S. A. Sadat, D. Haralson, M. Sahraei-Ardakani, Evaluation of Various Techniques to Warm-Start a Successive Linear Programming Algorithm for Solving the IV ACOPF, in: 2018 IEEE Power Energy Society General Meeting (PESGM), 2018, pp. 1-5.

[30] S. A. Sadat, M. Sahraei-Ardakani, Initializing Successive Linear Programming Solver for ACOPF using Machine Learning, in: 2020 IEEE North American Power Symposium (NAPS), 2020, pp. 1-6.

[31] Z. Yang, H. Zhong, Q. Xia, C. Kang, Solving opf using linear approximations: fundamental analysis and numerical demonstration, IET Generation, Transmission Distribution 11 (17) (2017) 4115-4125.

[32] Z. Yang, H. Zhong, Q. Xia, A. Bose, C. Kang, Optimal power flow based on successive linear approximation of power flow equations, IET Generation, Transmission Distribution 10 (14) (2016) 3654-3662.

[33] Z. Yang, A. Bose, H. Zhong, N. Zhang, Q. Xia, C. Kang, Optimal reactive power dispatch with accurately modeled discrete control devices: A successive linear approximation approach, IEEE Transactions on Power Systems 32 (3) (2017) 2435-2444.

[34] P. Fortenbacher, T. Demiray, Linear/quadratic programming-based optimal power flow using linear power flow and absolute loss approximations, International Journal of Electrical Power \& Energy Systems 107 (2019) 
680-689.

[35] A. A. Mohamed, B. Venkatesh, Line-wise optimal power flow using successive linear optimization technique, IEEE Transactions on Power Systems 34 (3) (2019) 2083-2092.

[36] L. Sampath, B. V. Patil, H. Gooi, J. Maciejowski, K. Ling, A trust-region based sequential linear programming approach for ac optimal power flow problems Electric Power Systems Research 165 (2018) $134-143$. doi:https://doi.org/10.1016/j.epsr.2018.09.002

URL http://www.sciencedirect.com/science/article/pii/ S0378779618302955

[37] R. Byrd, N. Gould, J. Nocedal, R. Waltz, An algorithm for nonlinear optimization using linear programming and equality constrained subproblems, Mathematical Programming 100 (1) (2004) 27-48, cited By 70. doi:10.1007/s10107-003-0485-4

[38] S. Mhanna, P. Mancarella, An exact sequential linear programming algorithm for the optimal power flow problem, IEEE Transactions on Power Systems (2021) 1-1doi : 10.1109/TPWRS . 2021.3097066

[39] S. A. Sadat, Evaluating the Performance of Various ACOPF Formulations Using Nonlinear Interior-Point Method, 2021 IEEE International Smart Cities Conference (ISC2) (2021) 1-idoi:10.1109/ISC253183.2021. 9562928

[40] C. Coffrin, H. L. Hijazi, P. Van Hentenryck, The qc relaxation: A theoretical and computational study on optimal power flow, IEEE Transactions on Power Systems 31 (4) (2016) 3008-3018. doi:10.1109/TPWRS. 2015. 2463111

[41] A. Forsgren, P. E. Gill, E. Wong, Active-set methods for convex quadratic programming, arXiv preprint ArXiv:1503.08349 (2015).

[42] A. Castillo, P. Lipka, J. P. Watson, S. S. Oren, R. P. O'Neill, A successive linear programming approach to solving the iv-acopf, IEEE Transactions on Power Systems 31 (4) (2016) 2752-2763. doi:10.1109/TPWRS. 2015.2487042

[43] ARPAE Grid Optimization Competition, SCOPF Problem Formulation: Challenge 1. U.S. Department of Energy (2019) 18. URL https://gocompetition.energy.gov/sites/default/ files/SCOPF_Problem_Formulation__Challenge_1_20190412. pdf

[44] ARPAE Grid Optimization Competition, GO Competition Challenge 1 Datasets U.S. Department of Energy (2019) 1.

URL https://gocompetition.energy.gov/challenges/22/ datasets 